\title{
Poor Prognosis of Grade 2 Spread Through Air Spaces in Neuroendocrine Tumors
}

\author{
Mincheol Chae, M.D. ${ }^{1}$, Sukki Cho, M.D., Ph.D. ${ }^{1,2}$, Jin-Haeng Chung, M.D., Ph.D. ${ }^{3,4}$, Sungwon Yum, B.S. ${ }^{1}$, \\ Kwhanmien Kim, M.D., Ph.D. ${ }^{1,2}$, Sanghoon Jheon, M.D., Ph.D.,2 \\ 'Department of Thoracic and Cardiovascular Surgery, Seoul National University Bundang Hospital, Seongnam; ${ }^{2}$ Department of Thoracic and Cardiovascular \\ Surgery, Seoul National University College of Medicine, Seoul; ${ }^{3}$ Department of Pathology and Translational Medicine, Seoul National University Bundang Hospital, \\ Seongnam; ${ }^{4}$ Department of Pathology, Seoul National University College of Medicine, Seoul, Korea
}

\section{ARTICLE INFO}

Received September 1, 2021

Revised December 8, 2021

Accepted December 29, 2021

Corresponding author

Sukki Cho

Tel $82-31-787-7132$

Fax 82-31-787-4050

E-mail skcho@snubh.org

ORCID

https://orcid.org/0000-0002-9309-8865
Background: Spread through air spaces (STAS) has recently emerged as a prognostic factor in lung adenocarcinoma, but little is known about the association of STAS and its grade with recurrence in neuroendocrine tumors (NETs). This study investigated the prognostic effect of STAS grade in NETs after curative resection.

Methods: Seventy-seven patients were retrospectively reviewed, including 9 with typical carcinoid (TC), 6 with atypical carcinoid (AC), 26 with large cell neuroendocrine carcinoma (LCNEC), and 36 with small cell carcinoma (SCC). STAS was defined as the presence of floating tumor cells within air spaces in the lung parenchyma beyond the edge of the main tumor. STAS was classified as grade 1 or 2 depending on whether it was found within or beyond one $\times 10$ objective lens field away from the main tumor margin, respectively.

Results: Fifty-four patients (70\%) had STAS, including 22\% with TC, 50\% with AC, 69\% with LCNEC, and $86 \%$ with SCC. Patients with STAS had more nodal metastasis, lymphatic and vascular invasion, tumor necrosis, and tumor subtypes other than TC. Among STAS cases, grade 2 STAS was present in $33 \%$ of AC, $78 \%$ of LCNEC, and $87 \%$ of SCC. The 5 -year recurrence-free survival (RFS) rate was $81 \%, 63 \%$, and $35 \%$ in patients with no STAS, grade 1, and grade 2 STAS, respectively. Multivariate analysis found that grade 2 STAS was an independent negative prognostic factor for RFS.

Conclusion: Although STAS itself was not associated with a poor prognosis, grade 2 STAS was an independent negative prognostic factor for RFS.

Keywords: Spread through air spaces, Neuroendocrine tumors, Prognosis, Grade

\section{Introduction}

Neuroendocrine tumors (NETs) are divided into lowgrade (typical carcinoid [TC]), intermediate-grade (atypical carcinoid $[\mathrm{AC}]$ ), and high-grade (large cell neuroendocrine carcinoma [LCNEC] and small cell carcinoma [SCC]) according to their prognosis.

Spread through air spaces (STAS), which is defined as the spread of lung cancer tumor cells into air spaces in the lung parenchyma adjacent to the main tumor, is a newly recognized mechanism of recurrence in lung adenocarcinoma [1]. The prognostic effect of STAS in lung adenocarcinoma is well known, and it is particularly strong after limited resection [2]. Even in lung squamous cell carcino- ma, STAS has a prognostic effect after curative resection [3]. However, little is known regarding the association of STAS with recurrence in NETs.

STAS can be classified as no STAS, low-grade STAS, or high-grade STAS according to the number of clusters of STAS or tumor cells. High-grade STAS was found to be associated with a worse prognosis than no STAS or lowgrade STAS [4]. In pathology reports at our center, STAS has been reported in resected lung cancer specimens since 2008 and has been graded using a 2-tier system according to the distance from the edge of the tumor margin since 2011.

Therefore, this study was conducted to evaluate the prognostic effect of STAS grade in NETs after curative resection. 


\section{Methods}

\section{Patients}

This study is a retrospective review of a prospective lung cancer database. Patients enrolled in this study underwent curative resection from January 2011 to December 2018 and were diagnosed pathologically with NETs. All patients underwent complete resection with mediastinal node dissection. The tumors were staged pathologically according to the eighth edition of the TNM (tumor-node-metastasis) classification. Patients who received neoadjuvant therapy, had distant metastasis, or experienced incomplete resection were excluded. This study was approved by the Institutional Review Board of Seoul National University Bundang Hospital (B-1801-445-405). The requirement for informed consent was waived since it was a retrospective review.

\section{Histologic examination}

STAS was defined as the presence of floating tumor cells within air spaces in the lung parenchyma beyond the edge of the main tumor. The edge of the main tumor was defined as the outer border of the tumor, which is typically visualized using a low-power histologic examination. Since 2011, the extent of STAS has been graded using a 2-tier system according to the distance from the edge of tumor margin. When tumor clusters were present only within 1 field of a $\times 10$ objective lens $(2,500 \mu \mathrm{m}$ diameter $)$ away from the main tumor margin, STAS was graded as 1 , and if tumor clusters existed beyond the grade 1 area, STAS was graded as 2 (Fig. 1).

\section{Recurrence}

Patients were followed up every 6 months for the first 3 years and then every 8 months for an additional 2 years. The data analyzed in this study included the patient's history, physical examination findings, chest radiography, and tumor markers. Contrast-enhanced chest computed tomography (CT) was performed every 6 months for the first 3 years and then every 8 months thereafter. If recurrence was suspected, based on either newly presenting symptoms or scheduled tests, integrated positron emission tomography/CT (PET/CT) was performed. Even if patients showed no symptoms or abnormal findings in the scheduled tests, integrated PET/CT was performed approximately 1 year after curative resection. The final diagnosis of recurrence was confirmed by the histopathologic examination of sam-

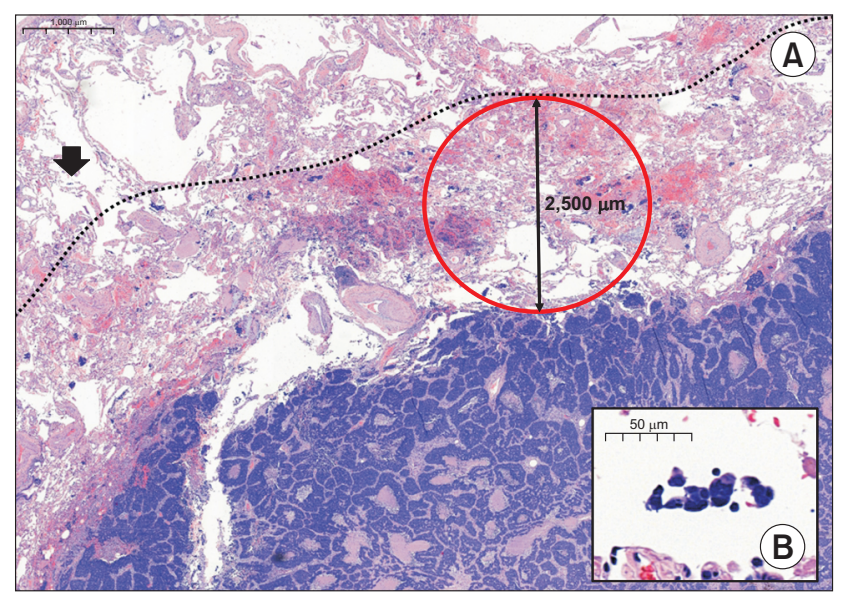

Fig. 1. Definition of extent of spread through air spaces (STAS) grading in histologic examination and typical case. (A) Definition of STAS grading; when tumor clusters existed within 1 field of a $\times 10$ objective lens $(2,500 \mu \mathrm{m}$ diameter: red circle) away from the edge of the main tumor, inside the dotted line, it was graded 1, and tumor clusters existing beyond the STAS 1 area were graded 2 ( $\times 15$ magnification). This case was STAS 2 tumor (black arrow; $\times 400$ magnification). (B) Typical case of STAS in small cell lung cancer ( $\times 50$ magnification)

ples obtained from surgery or biopsy. If it was impossible to diagnose recurrence histopathologically, recurrent malignancy was no longer suspected based on a clinical and radiologic follow-up period of at least 12 months with no evidence of active malignancy. Locoregional recurrence was defined as recurrence in the ipsilateral hemithorax, including the mediastinal lymph nodes, and distant metastasis was defined as recurrence in the contralateral hemithorax and extrathoracic organs.

\section{Statistical analysis}

The Student t-test and analysis of variance were used to compare the distributions of continuous data, and the Fisher exact test or the chi-square test was used to compare the frequencies of categorical measures among different groups. The length of overall survival (OS) was defined as the interval between the date of surgical resection and the date of either death or the last follow-up. Recurrence-free survival (RFS) was defined as the interval between the date of surgical resection and the date of the first recurrence or the last follow-up. An observation was censored at the last follow-up session when the patient was alive with recurrence-free status. The Kaplan-Meier log-rank test was used for univariate analysis, and a Cox proportional-hazards model was used for multivariate analysis. A forward stepwise selection procedure was implemented with a p-value 
threshold of 0.05 for inclusion in the multivariate analysis. Statistical significance was accepted when the p-value was less than 0.05. All data were analyzed using PASW SPSS ver. 18.0 (SPSS Inc., Chicago, IL, USA).

\section{Results}

\section{Characteristics of the patients}

NETs were found in a total of 77 patients, including 9 (12\%) with TC, 6 (8\%) with AC, 26 (34\%) with LCNEC, and
36 (47\%) with SCC. Of the 77 enrolled patients, 60 (78\%) were men, and their mean age was 65 years. There were 58 ever-smokers (75\%) and 19 never-smokers (25\%). The surgical approach was video-assisted thoracic surgery in 64 patients $(83 \%)$. Limited resection, including wedge resection $(n=10)$ and segmentectomy $(n=4)$, was performed in 14 patients $(18 \%)$. The pathological $\mathrm{T}$ stage was $\mathrm{T} 1$ in $39 \mathrm{pa}-$ tients (51\%), T2 in 26 patients (34\%), and T3 in 12 patients (16\%), and the $\mathrm{N}$ stage was N0 in 46 patients (60\%), N1 in 22 patients (29\%), and N2 in 9 patients (11\%).

Table 1. Characteristics of surgically resected patients with neuroendocrine tumors

\begin{tabular}{|c|c|c|c|c|}
\hline \multirow{2}{*}{ Characteristic } & \multicolumn{4}{|c|}{ Study cohort } \\
\hline & All patients $(n=77)$ & STAS (-) $(n=23)$ & STAS $(+)(n=54)$ & p-value \\
\hline Age $(y r)$ & $65.3 \pm 11.8$ & $60.1 \pm 16.9$ & $65.3 \pm 11.5$ & 0.126 \\
\hline Sex & & & & 0.580 \\
\hline Male & $60(77.9)$ & $17(28.3)$ & $43(71.7)$ & \\
\hline Female & $17(22.1)$ & $6(35.3)$ & $11(64.7)$ & \\
\hline Smoking & & & & 0.179 \\
\hline Never-smoker & $19(24.7)$ & $8(42.1)$ & $11(57.9)$ & \\
\hline Ever-smoker & $58(75.3)$ & $15(25.9)$ & $43(74.1)$ & \\
\hline Chronic obstructive pulmonary disease & & & & 0.594 \\
\hline Absent & $68(88.3)$ & $21(30.9)$ & $47(69.1)$ & \\
\hline Present & $9(11.7)$ & $2(22.2)$ & $7(77.8)$ & \\
\hline Tumor size $(\mathrm{cm})$ & $3.0 \pm 1.5$ & $2.8 \pm 1.3$ & $2.8 \pm 1.4$ & 0.969 \\
\hline Maximum standardized uptake value & $8.1 \pm 4.3$ & $8.1 \pm 4.3$ & $7.5 \pm 3.5$ & 0.671 \\
\hline Surgical approach & & & & 0.159 \\
\hline Video-assisted thoracic surgery & $64(83.1)$ & $17(26.6)$ & $47(73.4)$ & \\
\hline Open & $13(16.9)$ & $6(46.2)$ & $7(53.8)$ & \\
\hline Surgical extent & & & & 0.748 \\
\hline Limited resection & $14(18.2)$ & $5(35.7)$ & $9(64.3)$ & \\
\hline Lobar resection & $63(81.8)$ & $18(28.6)$ & $45(71.4)$ & \\
\hline pT & & & & 0.540 \\
\hline pT1 & $39(50.6)$ & $13(33.3)$ & $26(66.7)$ & \\
\hline pT2 & $26(33.8)$ & $8(30.8)$ & $18(69.2)$ & \\
\hline pT3 & $12(15.6)$ & $2(16.7)$ & $10(83.3)$ & \\
\hline Subtype & & & & 0.001 \\
\hline Typical carcinoid & $9(11.7)$ & $7(77.8)$ & $2(22.2)$ & \\
\hline Atypical carcinoid & $6(7.8)$ & $3(50.0)$ & $3(50.0)$ & \\
\hline Large cell neuroendocrine carcinoma & $26(33.8)$ & $8(30.8)$ & $18(69.2)$ & \\
\hline Small cell carcinoma & $36(46.8)$ & $5(13.9)$ & $31(86.1)$ & \\
\hline $\mathrm{pN}$ & & & & 0.008 \\
\hline $\mathrm{pN} 0$ & $46(59.7)$ & $19(41.3)$ & $27(58.7)$ & \\
\hline $\mathrm{pN}+$ & $31(40.3)$ & $4(12.9)$ & $27(87.1)$ & \\
\hline Lymphatic invasion & $44(57.1)$ & $5(11.4)$ & $39(88.6)$ & 0.001 \\
\hline Vascular invasion & $41(53.9)$ & $5(12.2)$ & $36(87.8)$ & 0.001 \\
\hline Necrosis & $54(70.1)$ & $11(20.4)$ & $43(79.6)$ & 0.005 \\
\hline Visceral pleural invasion & $19(24.7)$ & $3(15.8)$ & $16(84.2)$ & 0.122 \\
\hline Perineural invasion & $4(5.2)$ & 0 & $4(100.0)$ & 0.180 \\
\hline Adjuvant therapy & $48(62.3)$ & $8(34.8)$ & $40(74.1)$ & 0.001 \\
\hline
\end{tabular}

Values are presented as mean \pm standard deviation or number (\%).

STAS, spread through air spaces. 


\section{Prevalence of spread through air spaces}

STAS was present in 54 patients (70\%), including $22 \%$ of those with TC, $50 \%$ of those with AC, $69 \%$ of those with LCNEC, $86 \%$ of those with SCC. STAS was most frequently observed in patients with SCC. A comparison between the characteristics of STAS (-) and STAS (+) patients is shown in Table 1. STAS (+) patients had more nodal metastasis, lymphatic invasion (LI), vascular invasion (VI), and tumor necrosis. However, sex, age, smoking status, tumor size, maximum standardized uptake value on PET/CT, surgical extents, and visceral pleural invasion (VPI) were not significantly different between the 2 groups.

STAS was classified as grade 1 or grade 2 according to our institutional protocol. Grade 1 STAS was found in 12 patients $(16 \%)$ and grade 2 STAS was found in 42 patients (55\%). Among patients with STAS, grade 2 STAS was present in $0 \%$ of patients with TC, $33 \%$ of those with AC, $78 \%$ of those with LCNEC, and $87 \%$ of those with SCC. In a comparison of clinicopathologic variables between patients with grade 1 and grade 2 STAS, old age, ever-smoker status, and the SCC subtype were significantly more common among patients with grade 2 STAS (Table 2).

\section{Survival analysis}

The median follow-up duration was 34 months (interquartile range, 23-59 months). Twenty-six patients (34\%)

Table 2. Characteristics of patients with neuroendocrine tumors and grade 1 or grade 2 STAS

\begin{tabular}{|c|c|c|c|}
\hline \multirow{2}{*}{ Characteristic } & \multicolumn{3}{|c|}{ Study cohort } \\
\hline & STAS (grade 1) (n=12) & STAS (grade 2$)(n=42)$ & p-value \\
\hline Age (yr) & $58.9 \pm 17.8$ & $67.1 \pm 8.3$ & 0.028 \\
\hline Sex & & & 0.652 \\
\hline Male & $9(20.9)$ & $34(79.1)$ & \\
\hline Female & $3(27.3)$ & $8(72.3)$ & \\
\hline Smoking & & & 0.038 \\
\hline Never-smoker & $5(45.5)$ & $6(54.5)$ & \\
\hline Ever-smoker & $7(16.3)$ & $36(83.7)$ & \\
\hline Chronic obstructive pulmonary disease & & & 0.130 \\
\hline Absent & $12(25.5)$ & $35(74.5)$ & \\
\hline Present & 0 & $7(100.0)$ & \\
\hline Tumor size $(\mathrm{cm})$ & $2.3 \pm 0.7$ & $3.0 \pm 1.6$ & 0.146 \\
\hline Maximum standardized uptake value & $7.1 \pm 3.9$ & $7.7 \pm 3.4$ & 0.650 \\
\hline Surgical approach & & & 0.130 \\
\hline Video-assisted thoracic surgery & $12(25.5)$ & $35(74.5)$ & \\
\hline Open & 0 & $7(100.0)$ & \\
\hline pT & & & 0.319 \\
\hline pT1 & $8(30.8)$ & $18(69.2)$ & \\
\hline pT2 & $3(16.7)$ & $15(83.3)$ & \\
\hline pT3 & $1(10.0)$ & $9(90.0)$ & \\
\hline Subtype & & & 0.007 \\
\hline Typical carcinoid & $2(100.0)$ & 0 & \\
\hline Atypical carcinoid & $2(66.7)$ & $1(33.3)$ & \\
\hline Large cell neuroendocrine carcinoma & $4(22.2)$ & $14(77.8)$ & \\
\hline Small cell carcinoma & $4(12.9)$ & $27(87.1)$ & \\
\hline $\mathrm{pN}$ & & & 1.000 \\
\hline $\mathrm{pNO}$ & $6(22.2)$ & $21(77.8)$ & \\
\hline $\mathrm{pN}+$ & $6(22.2)$ & $21(77.8)$ & \\
\hline Lymphatic invasion & $8(20.5)$ & $31(79.5)$ & 0.626 \\
\hline Vascular invasion & $8(22.2)$ & $28(77.8)$ & 1.000 \\
\hline Necrosis & $8(18.6)$ & $35(81.4)$ & 0.206 \\
\hline Visceral pleural invasion & $4(25.0)$ & $12(75.0)$ & 0.750 \\
\hline Perineural invasion & $1(25.0)$ & $3(75.0)$ & 0.890 \\
\hline Adjuvant therapy & $6(50.0)$ & $34(81.0)$ & 0.057 \\
\hline
\end{tabular}

Values are presented as mean \pm standard deviation or number (\%).

STAS, spread through air spaces. 
died during follow-up, including 18 cancer-related deaths and 8 non-cancer-related deaths. Recurrence occurred in 30 patients (39\%), with locoregional recurrence in 5 patients and distant metastasis in 25 patients. The 5 -year RFS, OS, and cancer-specific survival rates were $54 \%, 64 \%$, and $72 \%$, respectively. The univariate analysis found that ever-smoker status; the LCNEC and SCC subtypes; nodal metastasis; the presence of LI, VI, necrosis, and perineural invasion; the presence of STAS; and grade 2 STAS were negative prognostic factors for RFS. In the multivariate analysis, grade 2 STAS was an independent negative prognostic factor for RFS (Table 3).

In the 23 patients without STAS, there was recurrence in 4 patients (17\%) and death in 4 patients (17\%). In contrast, of the 54 patients with STAS, 22 (41\%) died and $26(43 \%)$ experienced recurrence. Recurrence occurred in 4 (17\%), 3 (25\%), and 23 (55\%) patients with no STAS, grade 1 STAS, and grade 2 STAS, respectively. The proportion of recurrence was significantly different between the 2 groups $(\mathrm{p}=0.001)$. The 5 -year RFS rate was $81 \%, 63 \%$, and $35 \%$ in patients with no STAS and in those with grade 1 and grade 2 STAS, respectively $(\mathrm{p}=0.002)$ (Fig. $2 \mathrm{~A}$ ). The 5 -year RFS rate was $74 \%$ and $35 \%$ in patients with no STAS or grade 1 and grade 2 STAS, respectively ( $\mathrm{p}=0.001$ ) (Fig. 2B). In an analysis limited to patients with LCNEC and SCC, the 5 -year RFS rate was $66 \%$ and $31 \%$ in patients with no STAS or grade 1 STAS and grade 2 STAS, respectively $(\mathrm{p}=0.021)$.

\section{Discussion}

This study showed that STAS positivity was much more common in NETs than has been reported for other tumor types. STAS was closely related with subtype (SCC), nodal metastasis, LI, VI, and necrosis, which are known prognostic factors of NETs. Although STAS itself did not show an independent association with patients' prognoses, grade 2 STAS was an independent prognostic factor for RFS in NETs.

STAS was established as a fourth category defining invasion of adenocarcinomas in the World Health Organization classification, in addition to lepidic partition, stroma infiltration, and adjacent structural infiltration [5]. Since Travis et al. [5] demonstrated using 3-dimensional reconstructions that STAS tumor cells can attach to alveolar walls, rather than appearing free-floating, it has seemed that the debate on whether STAS is an artifact is over [6]. However, although STAS has been researched in lung adenocarcinoma and squamous cell carcinoma, there have been few reports about STAS in NETs.

In our study, STAS positivity was more common than has been reported in previous studies, with positivity rates of $50 \%$ in AC, $69 \%$ in LCNEC, and $86 \%$ in SCC, in contrast to the corresponding rates of $37 \%, 46 \%$, and $43 \%$, respectively, reported by Aly et al. [7]. However, Altinay et al. [8] reported a similar prevalence of STAS in NETs to our

Table 3. Univariate analysis of recurrence-free survival

\begin{tabular}{|c|c|c|c|c|}
\hline \multirow{2}{*}{ Characteristic } & \multicolumn{2}{|c|}{ Univariable analysis } & \multicolumn{2}{|c|}{ Multivariable analysis } \\
\hline & $\operatorname{HR}(95 \% \mathrm{Cl})$ & p-value & $\mathrm{HR}(95 \% \mathrm{Cl})$ & p-value \\
\hline Age & $1.030(0.998-1.064)$ & 0.063 & & \\
\hline Sex (male/female) & $2.030(0.769-5.360)$ & 0.153 & & \\
\hline Smoking (ever/never) & $2.700(1.017-7.164)$ & 0.046 & $2.188(0.560-8.553)$ & 0.260 \\
\hline COPD (present/absent) & $1.020(0.309-3.371)$ & 0.974 & & \\
\hline Tumor size & $1.026(0.752-1.400)$ & 0.870 & & \\
\hline SUVmax & $1.020(0.908-1.146)$ & 0.735 & & \\
\hline Approach (VATS/open) & $0.679(0.290-1.592)$ & 0.374 & & \\
\hline pT $(2 / 1)$ & $1.050(0.463-2.381)$ & 0.907 & & \\
\hline pT (3/1) & $1.263(0.459-3.473)$ & 0.651 & & \\
\hline Subtype (SCC+LCNEC/TC+AC) & $10.786(1.465-79.412)$ & 0.020 & $1.510(0.154-14.784)$ & 0.723 \\
\hline pN (positive/negative) & $3.370(1.614-7.041)$ & 0.001 & $2.055(0.874-4.832)$ & 0.099 \\
\hline LI (presence/absence) & $5.055(1.928-13.257)$ & 0.001 & $2.253(0.784-6.479)$ & 0.132 \\
\hline VI (presence/absence) & $4.481(1.824-11.012)$ & 0.001 & $2.640(0.999-6.977)$ & 0.050 \\
\hline Necrosis (presence/absence) & $3.555(1.235-10.234)$ & 0.019 & $1.819(0.440-7.516)$ & 0.409 \\
\hline Perineural invasion (presence/absence) & $5.332(1.767-16.089)$ & 0.003 & $1.247(0.376-4.137)$ & 0.719 \\
\hline VPI (presence/absence) & $1.776(0.824-3.829)$ & 0.142 & & \\
\hline STAS (grade 2/none+grade 1 ) & $4.184(1.779-9.838)$ & 0.001 & $3.109(1.159-8.341)$ & 0.024 \\
\hline
\end{tabular}

$\mathrm{HR}$, hazard ratio; $\mathrm{Cl}$, confidence interval; COPD, chronic obstructive pulmonary disease; SUVmax, maximum standardized uptake value; VATS, video-assisted thoracic surgery; SCC, small cell carcinoma; LCNEC, large cell neuroendocrine carcinoma; TC, typical carcinoid; AC, atypical carcinoid; LI, lymphatic invasion; VI, vascular invasion; VPI, visceral pleural invasion; STAS, spread through air spaces. 

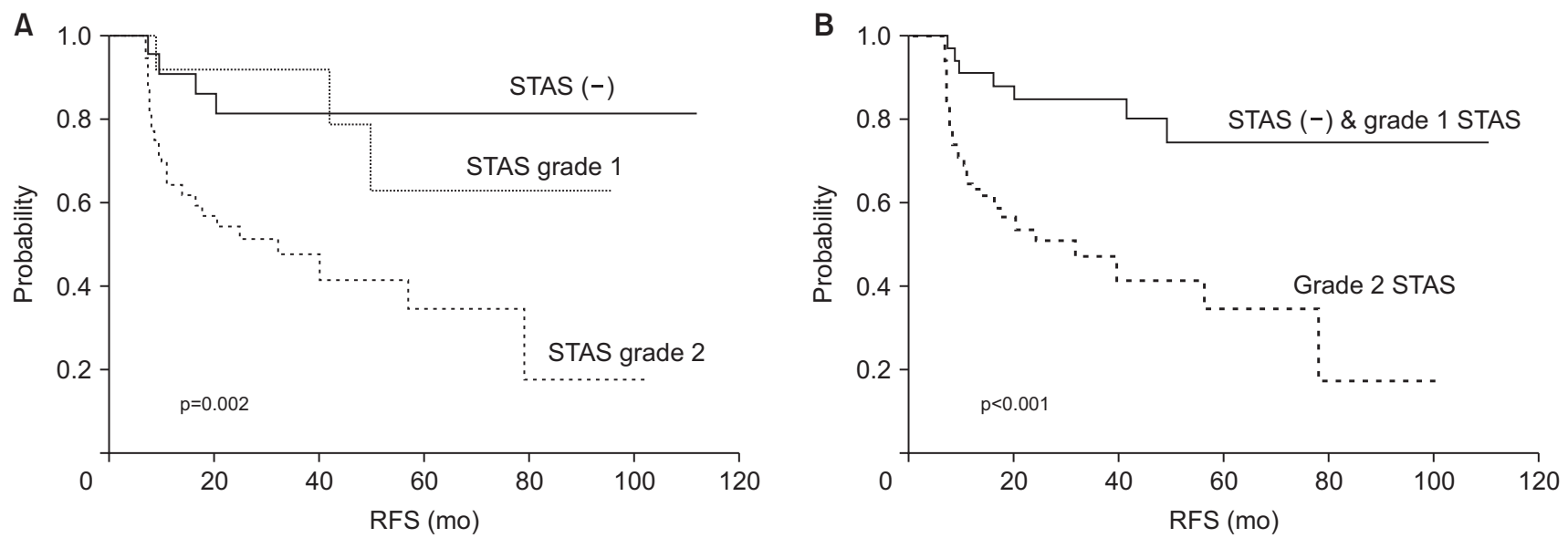

Fig. 2. Recurrence-free survival. The 5-year recurrence-free survival (RFS) rate was $81 \%$ without spread through air spaces (STAS), $63 \%$ with grade 1 STAS, and 35\% with grade 2 STAS (A). The 5 -year RFS rate was $74 \%$ and $35 \%$ in patients with no STAS or grade 1 and grade 2 STAS, respectively $(\mathrm{B})$.

results. Since few studies have investigated STAS in NETs, the exact prevalence of STAS in NETs cannot be considered to have been conclusively established; however, it has been consistently found that STAS occurs more frequently in NETs than in other tumor types. The trend observed in this study of increasing prevalence of STAS from TC to AC, LCNEC, and SCC is consistent with the findings of other research groups.

STAS was significantly associated with other confounding poor prognostic features, and this correlation has been argued to be the only reason why STAS is associated with a poor prognosis. In this study, STAS positivity was significantly associated with nodal metastasis, LI, VI, and necrosis, which were all identified as poor prognostic factors for RFS in the univariate analysis. However, STAS itself was not a risk factor for RFS in the univariate analysis. Since NETs are well known to be more aggressive tumors than adenocarcinoma or squamous cell carcinoma, STAS positivity was much more common in NETs than in lung cancers of other cell types, and most patients underwent lobar resection. As such, the presence of STAS itself cannot be considered a risk factor for recurrence. Therefore, we focused on STAS grading, hypothesizing that higher-grade STAS might be a risk factor for recurrence, instead of simple STAS positivity. Uruga et al. [4] performed a semiquantitative assessment of STAS, divided into low-grade STAS (1-4 cells or clusters of STAS) and high-grade STAS ( $>4$ single cells or clusters), and found that one-third of resected adenocarcinomas had high-grade STAS and that higher-grade STAS was predictive of worse RFS. In this study, the grading system was different from that of Uruga et al. [4], as the extent of STAS was graded using a 2-tier system according to the distance from the edge of the tumor margin. If tumor clusters were present only within one $\times 10 \mathrm{ob}-$ jective lens field away from the main tumor margin, STAS was graded as 1 , and if they were found beyond the grade 1 area, STAS was graded as 2. Compared to patients with grade 1 STAS, those with grade 2 STAS did show statistically significant differences in any factors except the subtype of NETs. Furthermore, grade 2 STAS was an independently significant prognostic factor for RFS.

Several studies have defined STAS as the presence of tumor cells at a distance from the edge of the primary tumor in the first alveolar space, while others have defined the necessary distance as at least a few alveolar spaces. Warth et al. [2] defined limited STAS as a solid nest no more than 3 alveoli away from the main tumor and extensive STAS as a tumor nest more than 3 alveoli away from the tumor edge. Lu et al. [3] measured the distance between the tumor edge and the farthest STAS cluster, and reported that the median distance was $1.4 \mathrm{~mm}$ (range, $0.3-4.2 \mathrm{~mm}$ ) and 5 alveolar spaces (range, 1-15). In their results, the rate of any recurrence and lung cancer-specific death was similar between lung squamous cell carcinoma patients with limited and extensive STAS. Although further validation of these classifications is required, these results indicate that the extent and quantity of STAS might reflect the progression and invasion of the tumor.

No difference was found in this study between grade 1 and grade 2 STAS in terms of relationships with previously established prognostic factors such as nodal metastasis, LI, VI, and necrosis. However, regarding nodal metastasis, it is noteworthy that grade 2 STAS was associated with a higher frequency of N1 and N2 metastasis than grade 1 STAS, al- 
though the difference was statistically insignificant due to the small sample size. The $\mathrm{T}$ stage showed a similar pattern to nodal metastasis between grade 1 and 2 . Therefore, if LI, VI, and necrosis-as factors that showed a statistically significant difference between STAS (-) and STAS (+) tumors-were evaluated as continuous values, not as dichotomized variables, they might have shown a closer relationship with grade 2 STAS.

Tumor cells detach from the main tumor and migrate through the air spaces to distant alveolar walls, where they reattach through vessel co-option of pre-existing blood vessels, leading to intrapulmonary spread [6]. This may explain the worse RFS in patients with grade 2 STAS despite lobar resection.

This study has some limitations. First, a small number of patients were retrospectively evaluated because there were relatively few surgically indicated patients with NETs, especially for SCC. Second, the grading system of STAS that was used in this study has not yet been standardized. However, we have used these criteria since 2011, and RFS was shorter in patients with grade 2 STAS than in those with grade 1 STAS, suggesting that the grading system used in this study might provide meaningful insights into patients' prognoses.

In conclusion, STAS was present more frequently in NETs than in tumors of other cell types in patients who underwent curative resection. Even though STAS positivity itself was not associated with poor prognoses, grade 2 STAS was an independent negative prognostic factor for RFS.

\section{Conflict of interest}

No potential conflict of interest relevant to this article was reported.

\section{ORCID}

Mincheol Chae: https://orcid.org/0000-0001-9280-8514
Sukki Cho: https://orcid.org/0000-0002-9309-8865

Jin-Haeng Chung: https://orcid.org/0000-0002-6527-3814

Sungwon Yum: https://orcid.org/0000-0002-9198-1021

Kwhanmien Kim: https://orcid.org/0000-0002-6581-2750

Sanghoon Jheon: https://orcid.org/0000-0001-9366-5981

\section{References}

1. Kadota K, Nitadori JI, Sima CS, et al. Tumor spread through air spaces is an important pattern of invasion and impacts the frequency and location of recurrences after limited resection for small stage $I$ lung adenocarcinomas. J Thorac Oncol 2015;10:806-14.

2. Warth A, Muley T, Kossakowski CA, et al. Prognostic impact of intra-alveolar tumor spread in pulmonary adenocarcinoma. Am J Surg Pathol 2015;39:793-801.

3. Lu S, Tan KS, Kadota K, et al. Spread through air spaces (STAS) is an independent predictor of recurrence and lung cancer-specific death in squamous cell carcinoma. J Thorac Oncol 2017;12:223-34.

4. Uruga H, Fujii T, Fujimori S, Kohno T, Kishi K. Semiquantitative assessment of tumor spread through air spaces (STAS) in early-stage lung adenocarcinomas. J Thorac Oncol 2017;12:1046-51.

5. Travis WD, Brambilla E, Nicholson AG, et al. The 2015 World Health Organization classification of lung tumors: impact of genetic, clinical and radiologic advances since the 2004 classification. J Thorac Oncol 2015;10:1243-60.

6. Yagi Y, Aly RG, Tabata K, et al. Three-dimensional histologic, immunohistochemical, and multiplex immunofluorescence analyses of dynamic vessel co-option of spread through air spaces in lung adenocarcinoma. J Thorac Oncol 2020;15:589-600.

7. Aly RG, Rekhtman N, Li X, et al. Spread through air spaces (STAS) is prognostic in atypical carcinoid, large cell neuroendocrine carcinoma, and small cell carcinoma of the lung. J Thorac Oncol 2019;14: 1583-93.

8. Altinay S, Metovic J, Massa F, et al. Spread through air spaces (STAS) is a predictor of poor outcome in atypical carcinoids of the lung. Virchows Arch 2019;475:325-34. 